\title{
Accurate three-dimensional tracking method in bistatic forward scatter radar
}

\author{
Cheng Hu, Tao Zeng and Chao Zhou
}

\begin{abstract}
Accurate three-dimensional (3D) tracking in bistatic forward scatter radar (BFSR) is a challenging problem because of absent range resolution and poor measurements. In this article, an accurate 3D tracking method of BFSR is proposed. Aiming to overcome the filter divergence caused by large initial state estimation error, firstly, an accurate initial state estimation approach is presented based on analytic derivation and Levenberg-Marquardt algorithm, which has the potential to improve the accuracy of initial state estimation. Furthermore, in order to reduce the computation cost of filtering process and speed up the filtering convergence rate, the accurate initial state estimation and extended Kalman filter algorithm in BFSR are combined to achieve a precise target 3D tracking. Finally, the proposed accurate tracking method is verified through comparative analysis of the simulation results.
\end{abstract}

Keywords: Forward scatter radar, 3D tracking, Initial state estimation, Filtering algorithm

\section{Introduction}

Because of the gain in bistatic RCS (up to 20-40 dB relative to monostatic RCS) in forward scatter region, bistatic forward scatter radar (BFSR) can effectively detect and track the target with low-speed or small RCS (including the stealth target) [1,2]. Its target tracking technology attracted more and more attention in recent years. An existing challenge of BFSR is parameter estimation of aerial [2-9] and ground [10-17] targets. This article focuses on parameter estimation of aerial target trajectory.

Systems using continuous quasi-harmonic probe signal have been proved to be most promising amongst various structures of BFSR [3-5]. In this case, measurements of echo Doppler shift $f_{\mathrm{d}}$, angle of arrival $\theta$, and elevation angle $\beta$ are usually used to obtain object parameter estimations (i.e., target position and velocity denoted as $\left.x_{k}, y_{k}, h, V_{x}, V_{y}\right)$. Due to the nonlinear relations between $f_{\mathrm{d}}, \theta, \beta$, and trajectory parameters,target parameter estimation in BFSR is a nonlinear optimization problem. To obtain five unknown variables (i.e., $x_{k}, y_{k}, h, V_{x}$, and $V_{y}$ ) using three known variables (i.e., $f_{\mathrm{d}}, \theta, \beta$ ), equations composed by groups of observations are needed. Hence,

\footnotetext{
* Correspondence: cchchb@gmail.com

Department of Electronic Engineering, Beijing Institute of Technology, Beijing 100081, China
}

object parameter estimation in BFSR is actually solving over-determined nonlinear equations. The optimization is achieved using nonlinear least squares algorithms, of which Gauss-Newton method has widely been used.

In previous research [3-7], Gauss-Newton method is adopted to solve the nonlinear equations. On this basis, the classical methods of target tracking using two-dimensional (2D) and three-dimensional (3D) BFSR are proposed, respectively, in $[4,6]$. The major problem is that Gauss-Newton method is not a "real-time" algorithm, since we have to collect $n$ measurements to implement it. To achieve higher data update rate and reduce computation in parameter estimation, extended Kalman filter (EKF) algorithm [18] is used. However, initial filtering value of EKF can only be obtained by solving over-determined nonlinear equations, which can never be avoided. In classical method, due to the special geometry of FSR system, the initial value estimation by Gauss-Newton method requires high-precision Doppler shift measurement and angle measurement, which are unavailable due to the limitation of antenna size and accumulation time. Therefore, Gauss-Newton method is easy to cause a large error of initial value estimation and filtering divergence.

In this article, based on analytical derivation and Levenberg-Marquardt (LM) [19,20] algorithm, a new

\section{Springer}


approach of initial state estimation is presented, which can improve the accuracy of initial state estimation without high-precise target parameters measurement. Hereafter, the accurate initial state estimation and EKF algorithm are combined to achieve fast convergence and high accuracy. Simulation results prove that accurate target trajectory parameters estimation can be obtained by this method before the target crossing the baseline.

The remainder of this article is organized as follows: Section 1 briefly describes the development of forward scatter radar, pointing out the existing problems of air target parameter estimation and focus of this article; Section 2 defines the geometry of 3D forward scatter radar, the target motion model and system observation model; Section 3 introduces the proposed tracking method for forward scatter radar in details; Section 4 verifies the validity of accurate tracking method through comparative analysis of the simulation results; Conclusions are drawn in Section 5.

\section{System modeling}

\section{Geometry and system implementation of 3D BFSR}

Geometry of 3D BFSR is shown in Figure 1. $x, y, z$ are Cartesian coordinates. $\mathrm{Re}, \mathrm{Tr}$, and $\mathrm{Tg}$ denote the positions of receiver, transmitter, and target, respectively. $\psi$ is flight-path angle (the trajectory inclination angle towards the baseline), $\theta$ is the target azimuth angle, $\beta$ is the target elevation angle, $h$ is the target altitude, $b$ is the distance between transmitter and receiver (i.e., the baseline length), $A B$ is the target trajectory, $C D$ is the projection of target trajectory in horizontal plane.

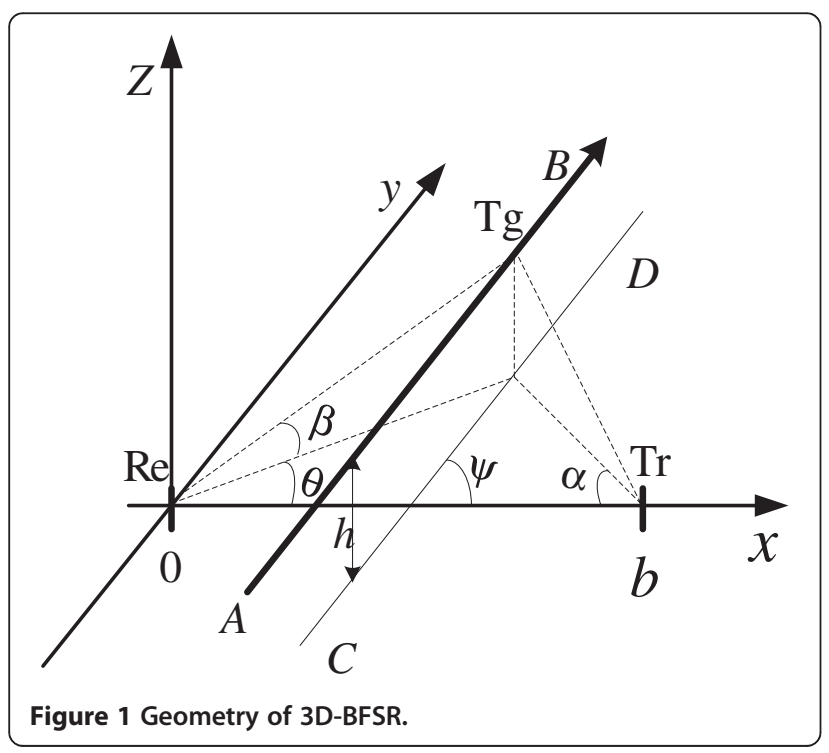

System settings meet the following principles:

1) In the transmitter position, there is a wide beam antenna illuminating the whole forward scatter region with a quasi-harmonic signal.

2) In the receiver position, there is a multi-beam antenna to illuminate the forward scatter region.

3) There is no block in line of sight between the transmitting and receiving antenna.

\section{Target model}

Assuming the target crosses the baseline near the midpoint in a constant speed with linear trajectory, target state equation can be written as

$$
X(k+1)=\Phi X(k)+G v(k)
$$

Assuming the target flies in a constant altitude (i.e., the longitudinal velocity is zero), target's state vector can be denoted by $X(k)=\left[x_{k}, y_{k}, h V_{x}, V_{y}\right]$, where $T$ means sampling interval, $v(k)$ is Gaussian white noise process with zero mean. $x_{k}, y_{k}, h, V_{x}, V_{y}$ are the values of target Cartesian coordinates at $k$ th discrete time instant and their derivatives. State transition matrix $\Phi$ and noise distribution matrix $G$ are, respectively, written as

$$
\Phi=\left[\begin{array}{ccccc}
1 & 0 & 0 & T & 0 \\
0 & 1 & 0 & 0 & T \\
0 & 0 & 1 & 0 & 0 \\
0 & 0 & 0 & 1 & 0 \\
0 & 0 & 0 & 0 & 1
\end{array}\right] \quad G=\left[\begin{array}{ccc}
\frac{T^{2}}{2} & 0 & 0 \\
0 & \frac{T^{2}}{2} & 0 \\
0 & 0 & \frac{T^{2}}{2} \\
T & 0 & 0 \\
0 & T & 0
\end{array}\right]
$$

\section{Observation model}

Assuming observation vectors measured from first to $k$ th instant are

$$
\left[\left(f_{d 1}, \theta_{1}, \beta_{1}\right), \ldots,\left(f_{d k}, \theta_{k}, \beta_{k}\right)\right]^{T}
$$

where $f_{d k}, \theta, \beta_{k}$ are observations of the Doppler frequency shift, echo azimuth angle, and elevation angle.

Measurement equation can be written as

$$
\overrightarrow{Z_{k}}=h\left(\overrightarrow{x_{k}}\right)+\Delta \overrightarrow{Z_{k}}
$$




$$
\begin{aligned}
h\left(\overrightarrow{x_{k}}\right) & =\left[\begin{array}{l}
f_{d k} \\
\theta_{k} \\
\beta_{k}
\end{array}\right] \\
& =\left[\begin{array}{l}
-\frac{1}{\lambda}\left[\frac{x_{k} V_{x}+y_{k} V_{y}}{\sqrt{x_{k}^{2}+y_{k}^{2}+h^{2}}}+\frac{y_{k} V_{y}-\left(b-x_{k}\right) V_{x}}{\sqrt{\left(b-x_{k}\right)^{2}+y_{k}^{2}+h^{2}}}\right] \\
\arctan \left(\frac{y_{k}}{x_{k}}\right) \\
\arctan \frac{h}{\sqrt{x_{k}^{2}+y_{k}^{2}}}
\end{array}\right]
\end{aligned}
$$

$\vec{Z}_{k}$ is the observation vector of $k$ th instant, which is denoted by $\left(f_{d k}, \theta, \beta_{k}\right) . \vec{X}_{k}$ is state vector of $k$ th instant, denoted by $\left[x_{k}, y_{k}, h_{k}, V_{x}, V_{y}\right]$. The measurement noise denoted by $\Delta \vec{Z}_{k}$ is Gaussian white noise with zero mean, whose noise variances are $\sigma_{f}^{2}, \sigma_{\theta}^{2}, \sigma_{\beta}^{2}$, respectively.

\section{Accurate 3D tracking in forward scatter radar}

Because of the special geometry of forward scatter radar, parameters (i.e., Doppler shift, azimuth angle, elevation angle) cannot directly be measured when the target crosses the baseline, resulting in a large estimation error and seriously decreasing the tracking precision. In addition, the detection area of forward scatter radar is a very narrow region near the baseline where bistatic angle ranges from $135^{\circ}$ to $180^{\circ}$, indicating that target tracking is effectively implemented during a very short period of time. Therefore, a stable tracking trajectory should be obtained before the target crosses the baseline, which requires fast convergence of filtering algorithm. However, the large error of initial state estimation will decrease the convergence speed, even lead to filtering divergence. In summary, the initial state estimation is significant for target tracking in forward scatter radar, which extremely affects the filtering accuracy and convergence speed.

The classical method uses Gauss-Newton iteration to estimate the initial state of target. Gauss-Newton iteration is prone to singular matrix causing inaccurate computation results. And if the first value of iteration deviates far away from its true value, the results of iteration easily fall into local minimum and then cause large error of initial state estimation. To overcome these problems, based on analytical derivation and LM algorithm, a novel method is presented to obtain high-precise initial state estimation.

The basic principles of this new initial state estimation method are as follows.

According to the system observation equations, the observation vector $\left(f_{d n}, \theta_{n}, \beta_{n}\right)$ measured in $n$th discrete time instant could be written as

$$
\begin{aligned}
& f_{d n}=-\frac{1}{\lambda}\left[\frac{x_{n} V_{x}+y_{n} V_{y}}{\sqrt{x_{n}^{2}+y_{n}^{2}+h^{2}}}+\frac{y_{n} V_{y}-\left(b-x_{n}\right) V_{x}}{\sqrt{\left(b-x_{n}\right)^{2}+y_{n}^{2}+h^{2}}}\right] \\
& \theta_{n}=\arctan \frac{y_{n}}{x_{n}} \\
& \beta_{n}=\arctan \frac{h}{\sqrt{x_{n}^{2}+y_{n}^{2}}}
\end{aligned}
$$

Based on the observation vectors $\left(f_{d 1}, \theta_{1}, \beta_{1}\right),\left(f_{d 2}, \theta_{2}\right.$, $\left.\beta_{2}\right), \ldots\left(f_{d n}, \theta_{n}, \beta_{n}\right)$ of previous time instants, the target state vector $\left(x_{n}, y_{n}, V_{x}, V_{y}, h\right)$ in $n$th time instant can be obtained. The process is as follows:

From Equation (6), then

$$
\frac{y_{1}}{x_{1}}=\tan \theta_{1}, \ldots, \frac{y_{n}}{x_{n}}=\tan \theta_{n}
$$

The target motion model shows the relationship between $y_{1}$ and $y_{n}$ :

$$
\left\{\begin{array}{l}
y_{1}=y_{n}-(n-1) T V_{y} \\
x_{1}=x_{n}-(n-1) T V_{x}
\end{array}\right.
$$

Substituting Equation (9) into Equation(8), we have

$$
\frac{y_{n}-(k-1) T V_{y}}{x_{n}-(k-1) T V_{x}}=\tan \theta_{1}
$$

Based on Equations (8) and(10), then

$$
\left(\tan \theta_{n}-\tan \theta_{1}\right) x_{n}-(n-1) T V_{y}+\tan \theta_{1}(n-1) T V_{x}=0
$$

Similarly, we have

$$
\left(\tan \theta_{n}-\tan \theta_{2}\right) x_{n}-(n-2) T V_{y}+\tan \theta_{2}(n-2) T V_{x}=0
$$

And the following simultaneous equations

$$
\left\{\begin{array}{l}
\left(\tan \theta_{n}-\tan \theta_{1}\right) x_{n}-(n-1) T V_{y}+\tan \theta_{1}(n-1) T V_{x}=0 \\
\left(\tan \theta_{n}-\tan \theta_{2}\right) x_{n}-(n-2) T V_{y}+\tan \theta_{2}(n-2) T V_{x}=0
\end{array}\right.
$$

Let the coefficients of $x_{n}, V_{y}, V_{x}$ be $\left(a_{1}, a_{2}, a_{3}\right),\left(b_{1}, b_{2}\right.$, $b_{3}$ ), that is

$$
\begin{cases}a_{1}=\left(\tan \theta_{n}-\tan \theta_{1}\right) & b_{1}=\left(\tan \theta_{n}-\tan \theta_{2}\right) \\ a_{2}=-(n-1) T & b_{2}=-(n-2) T \\ a_{3}=\tan \theta_{1}(n-1) T & b_{3}=\tan \theta_{2}(n-2) T\end{cases}
$$


Then Equation (13) can be written as

$$
\left[\begin{array}{ll}
a_{1} & a_{3} \\
b_{1} & b_{3}
\end{array}\right]\left[\begin{array}{l}
x_{k} \\
V_{x}
\end{array}\right]=\left[\begin{array}{l}
-a_{2} \\
-b_{2}
\end{array}\right] V_{y}
$$

Equations above have solutions as follows

$$
\begin{aligned}
x_{n} & =\frac{a_{3} b_{2}-a_{2} b_{3}}{a_{1} b_{3}-a_{3} b_{1}} \cdot V_{y}=d_{n 1} V_{y} \\
V_{x} & =\frac{a_{2} b_{1}-a_{1} b_{2}}{a_{1} b_{3}-a_{3} b_{1}} \cdot V_{y}=d_{n 2} V_{y} \\
y_{n} & =\tan \theta_{k} \cdot d_{n 1} V_{y}=d_{n 3} V_{y}
\end{aligned}
$$

According to Equation (7), then

$$
h=\tan \beta_{n} \cdot \sqrt{x_{n}^{2}+y_{n}^{2}}
$$

Substituting Equations (16) and (18) into Equation (19), the relationship between $h$ and $V_{y}$ could be written as

$$
h=\tan \beta_{n} \sqrt{d_{n 1}^{2}+d_{n 3}^{2}}\left|V_{y}\right|=d_{n 4}\left|V_{y}\right|
$$

Substituting Equations (16), (17), (18), (20) into Equation (5), we can obtain the nonlinear equation as follows:

$$
\begin{aligned}
f_{d n} & +\frac{1}{\lambda}\left[\frac{\left(d_{n 1} d_{n 2}+d_{n 3}\right) V_{y}^{2}}{\sqrt{\left(d_{n 1}^{2}+d_{n 3}^{2}+d_{n 4}^{2}\right) V_{y}^{2}}}\right. \\
& \left.+\frac{d_{n 3} V_{y}^{2}-\left(b-d_{n 1} V_{y}\right) d_{n 2} V_{y}}{\sqrt{\left(b-d_{n 1} V_{y}\right)^{2}+d_{n 3}^{2} V_{y}^{2}+d_{n 4}^{2} V_{y}^{2}}}\right]=0
\end{aligned}
$$

Assuming that $N$ set of observations are used in initial state estimation, take $n=(N / 2+1): N$, we can get the following nonlinear equations
Solving Equation (22) by the LM algorithm can achieve the optimal solution of $V_{y}$, and $x_{n}, V_{x}, y_{n}, h$, which can also be obtained by Equation (16), (17), (18), and (20). Thus, the numerical solution of the target state $\left(x_{w}, y_{w}, h, V_{x}, V_{y}\right)$ in $n$th instant is acquired.

Because measurement noise is considerably large relative to the measurement data of azimuth angle, it is necessary to smooth primary measurements through polynomial fitting before the initial state estimation. Polynomial coefficients can be obtained by least square method, which can reduce the initial state estimation error by an order of magnitude.

In the stage of follow-up tracking, EKF algorithm is adopted. As mentioned earlier, the system state transition equation and measurement equation are as follows:

$$
\begin{aligned}
& X(k+1)=\Phi K(k)+G v(k) \\
& Z(k)=h(k, X(k))+W(k)
\end{aligned}
$$

where $h(k, X(k))$ denotes the measurement vector, measurement noise denoted by $W(k)$ is Gaussian white noise process with zero mean. Assuming the process noise covariance and measurement noise covariance at time $k$ are, respectively, expressed as $Q(k)$ and $R(k)$, then the steps of filtering are as follows:

State prediction:

$$
\hat{X}(k+1 \mid k)=\Phi X(k)
$$

Error covariance prediction:

$$
P(k+1 \mid k)=\Phi P(k \mid k) \Phi^{T}+Q(k)
$$

Measurements prediction:

$$
\hat{Z}(k+1 \mid k)=h[\hat{X}(k+1 \mid k)]
$$

Calculate residual covariance:

$$
S(k+1)=h_{X}(k+1) P(k+1 \mid k) h_{X}^{T}(k+1)+R(k+1)
$$

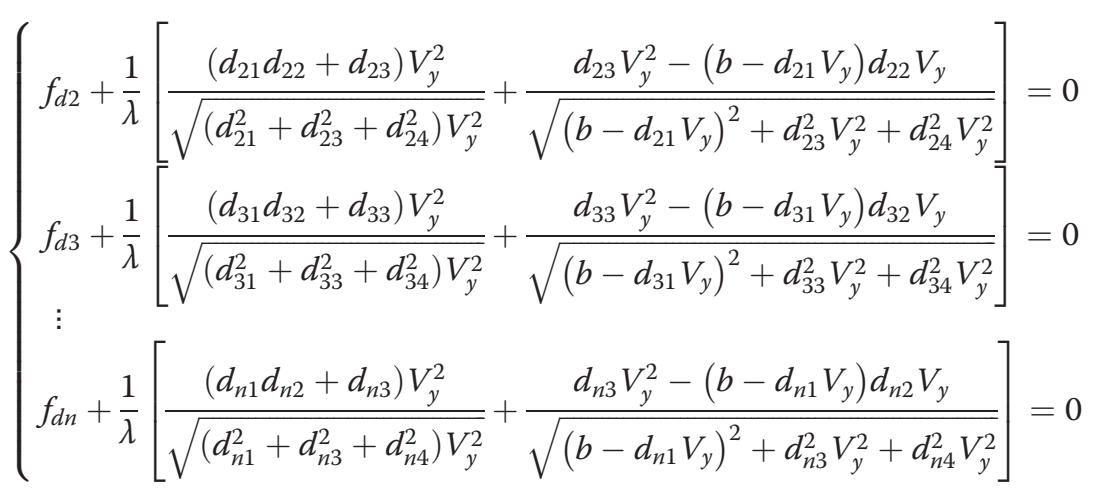




\section{Table 1 Simulation parameters}

\begin{tabular}{llll}
\hline Transmitted signal wavelength & $0.77 \mathrm{~m}$ & Standard deviation of process noise & 1 \\
Baseline length & $40 \mathrm{~km}$ & Target's velocity & Flight-path angle \\
Data update rate & $1 \mathrm{~Hz}$ & Target's initial $X$-coordinate & $150 \mathrm{~m} / \mathrm{s}$ \\
Standard deviation of Doppler shift measurements & $0.5 \mathrm{~Hz}$ & Target's initial Y-coordinate & $20 \mathrm{~km}$ \\
Standard deviation of azimuth measurements & $0.5^{\circ}$ & Target's initial H-coordinate & $-6 \mathrm{~km}$ \\
Standard deviation of elevation measurements & $0.5^{\circ}$ & $\mathrm{km}$
\end{tabular}

Calculate Kalman gain:

$$
K(k+1)=P(k+1 \mid k) h_{X}^{T}(k+1) S^{-1}(k+1)
$$

Update state:

$$
\begin{aligned}
\hat{X}(k+1 \mid k+1)= & \hat{X}(k+1 \mid k)+K(k+1) \\
& \times\{Z(k+1)-h[\hat{X}(k+1 \mid k)]\}
\end{aligned}
$$

Update error covariance:

$$
\begin{aligned}
P(k+1 \mid k+1)= & {\left[I-K(k+1) h_{X}(k+1)\right] P(k+1 \mid k) } \\
& \cdot\left[I-K(k+1) h_{X}(k+1)\right]^{T} \\
& -K(k+1) R(k+1) K^{T}(k+1)
\end{aligned}
$$

$h_{X}(k)$ in Equation (28) is the Jacobian matrix of $h(k, X$ $(k))$, which is

$$
\begin{aligned}
h(k, X(k))= & {\left[\begin{array}{c}
f_{D k}(X) \\
\alpha_{k}(X) \\
\beta_{k}(X)
\end{array}\right] } \\
= & {\left[\begin{array}{l}
-\frac{1}{\lambda}\left[\frac{x_{k} V_{x}+y_{k} V_{y}}{\sqrt{x_{k}^{2}+y_{k}^{2}+h^{2}}}+\frac{y_{k} V_{y}-\left(b-x_{k}\right) V_{x}}{\sqrt{\left(b-x_{k}\right)^{2}+y_{k}^{2}+h^{2}}}\right] \\
\alpha_{k}=\arctan \left(\frac{y_{k}}{x_{k}}\right) \\
\beta_{k}=\arctan \frac{h}{\sqrt{x_{k}^{2}+y_{k}^{2}}}
\end{array}\right] }
\end{aligned}
$$

Make

$$
R_{R}=\sqrt{x_{k}^{2}+y_{k}^{2}+h^{2}} R_{T}=\sqrt{\left(b-x_{k}\right)^{2}+y_{k}^{2}+h^{2}}
$$

Then

$$
\begin{aligned}
\frac{\partial f_{D k}}{\partial x_{k}}= & -\frac{1}{\lambda}\left(\frac{\left(y_{k}^{2}+h^{2}\right) V_{x}-x_{k} y_{k} V_{y}}{R_{R}^{3}}\right. \\
& \left.+\frac{\left(y_{k}^{2}+h^{2}\right) V_{x}+y_{k}\left(b-x_{k}\right) V_{y}}{R_{T}^{3}}\right) \\
\frac{\partial f_{D k}}{\partial y_{k}}= & -\frac{1}{\lambda}\left(\frac{\left(x_{k}^{2}+h^{2}\right) V_{y}-x_{k} y_{k} V_{x}}{R_{R}^{3}}\right. \\
& \left.+\frac{\left[\left(b-x_{k}\right)^{2}+h^{2}\right] V_{y}+y_{k}\left(b-x_{k}\right) V_{x}}{R_{T}^{3}}\right)
\end{aligned}
$$

\begin{tabular}{|c|c|c|c|c|c|c|}
\hline RMSE & & $x_{n} / \mathrm{m}$ & $y_{n} / \mathrm{m}$ & $h / \mathrm{m}$ & $V_{x} /(\mathrm{m} / \mathrm{s})$ & $V_{y} /(\mathrm{m} / \mathrm{s})$ \\
\hline $\begin{array}{l}\sigma_{f}=0.5 \\
\sigma_{\theta}=0.5\end{array}$ & Classical method & 2028.2 & 537.1 & 264.6 & 220.1 & 20.3 \\
\hline$\sigma_{\beta}=0.5$ & New method & 854.3737 & 255.1232 & 96.3750 & 2.1184 & 7.4299 \\
\hline $\begin{array}{l}\sigma_{f}=0.1 \\
\sigma_{\theta}=0.1\end{array}$ & Classical method & 773.0488 & 87.3466 & 83.5152 & 48.4883 & 9.8001 \\
\hline$\sigma_{\beta}=0.1$ & New method & 172.2670 & 50.3490 & 18.3618 & 0.9642 & 1.3896 \\
\hline
\end{tabular}

$$
\frac{\partial f_{D k}}{\partial h}=\frac{h}{\lambda}\left[\frac{x_{k} V_{x}+y_{k} V_{y}}{R_{R}^{3}}+\frac{y_{k} V_{y}-\left(b-x_{k}\right) V_{x}}{R_{T}^{3}}\right]
$$

$$
\frac{\partial f_{D k}}{\partial V_{x}}=-\frac{1}{\lambda}\left(\frac{x_{k}}{R_{R}}+\frac{x_{k}-b}{R_{T}}\right) \frac{\partial f_{D k}}{\partial V_{y}}=-\frac{V_{y}}{\lambda}\left(\frac{1}{R_{R}}+\frac{1}{R_{T}}\right)
$$

$\frac{\partial \alpha_{k}}{\partial x_{n}}=-\frac{y_{k}}{R_{R}^{2}} \frac{\partial \alpha_{k}}{\partial y_{n}}=\frac{x_{k}}{R_{R}^{2}} \frac{\partial \alpha_{k}}{\partial h}=\frac{\partial \alpha_{k}}{\partial V_{x}}=\frac{\partial \alpha_{k}}{\partial V_{y}}=0$

\section{Table 2 Simulation results of initial state estimation}


Table 3 Averaging run time of the three methods

\begin{tabular}{llll}
\hline & Classical method & New method & UKF algorithm \\
\hline $\begin{array}{l}\text { Average time } \\
\text { needed for a }\end{array}$ & 8.564927 & 0.14912884 & 0.32414323 \\
complete & & & \\
filtering (s) & & & \\
\hline
\end{tabular}

$$
\begin{aligned}
& \frac{\partial \beta_{k}}{\partial x_{k}}=-\frac{h x_{k}}{R_{R}^{3}+R_{R} h^{2}} \frac{\partial \beta_{k}}{\partial y_{k}}=-\frac{h y_{k}}{R_{R}^{3}+R_{R} h^{2}} \\
& \frac{\partial \beta_{k}}{\partial h}=\frac{\sqrt{x_{k}^{2}+y_{k}^{2}}}{x_{k}^{2}+y_{k}^{2}+h^{2}} \frac{\partial \beta_{k}}{\partial V_{x}}=\frac{\partial \beta_{k}}{\partial V_{y}}=0
\end{aligned}
$$

$h_{X}(k)$ is

$$
h_{X}(k)=\left[\begin{array}{lllll}
\frac{\partial f_{D k}(X)}{\partial x_{k}} & \frac{\partial f_{D k}(X)}{\partial y_{k}} & \frac{\partial f_{D k}(X)}{\partial h} & \frac{\partial f_{D k}(X)}{\partial V_{x}} & \frac{\partial f_{D k}(X)}{\partial V_{y}} \\
\frac{\partial \alpha_{k}(X)}{\partial x_{k}} & \frac{\partial \alpha_{k}(X)}{\partial y_{k}} & \frac{\partial \alpha_{k}(X)}{\partial h} & \frac{\partial \alpha_{k}(X)}{\partial V_{x}} & \frac{\partial \alpha_{k}(X)}{\partial V_{y}} \\
\frac{\partial \beta_{k}(X)}{\partial x_{k}} & \frac{\partial \beta_{k}(X)}{\partial y_{k}} & \frac{\partial \beta_{k}(X)}{\partial h} & \frac{\partial \beta_{k}(X)}{\partial V_{x}} & \frac{\partial \beta_{k}(X)}{\partial V_{y}}
\end{array}\right]_{X=\hat{X}(k \mid k)}
$$

\section{Simulations and analysis}

In order to verify the proposed accurate tracking algorithm, the parameters of FSR experiment system listed in references [3-6] are taken as the simulation parameters.

To prove the validity of the proposed method for initial state estimation, simulations are implemented with the parameters list in Table 1 using classical method and the new method, respectively, under the same parameters except standard deviations of Doppler shift, azimuth, and elevation denoted by $\left(\sigma_{f}, \sigma_{\theta}, \sigma_{\beta}\right)$. The results are averaging performed in terms of 100 independent implementations, as shown in Table 2, and their averaging run time are shown in Table 3.

Table 2 shows the initial statement estimation errors of classical method and the proposed method under the condition of different target measured parameters estimation accuracy. It is obviously that the statement estimation errors are affected by the estimation accuracy of target measured parameters. As can be seen, with high accuracy of target parameter measurement (i.e., $\sigma_{f}=0.1$ $\mathrm{Hz}, \sigma_{\theta}=0.1^{\circ}, \sigma_{\beta}=0.1^{\circ}$ ), estimation errors of the two methods are both within the error tolerance and the proposed method has higher accuracy. However, when measurement noise increases (i.e., $\sigma_{f}=0.5 \mathrm{~Hz}, \sigma_{\theta}=0.5^{\circ}$, $\left.\sigma_{\beta}=0.5^{\circ}\right)$, the initial state estimation error of classical method significantly increases, while that of proposed method is much smaller than the former. In particular, the velocity estimation error along the direction of the baseline is fairly small.

To compare the performances of the two methods more intuitively, simulations are implemented in case that initial state estimations obtained by the two methods are taken as the initial filtering value for EKF. The statement estimation results are shown in Figure 2. It can be seen that the initial statement estimation errors of Gauss-Newton iteration method are so large that the tracking results are divergent, while the EKF algorithm works very well with the initial statement obtained by the proposed method.

The averaged results performed in terms of 100 independent implementations are shown in Figure 3. In (a)

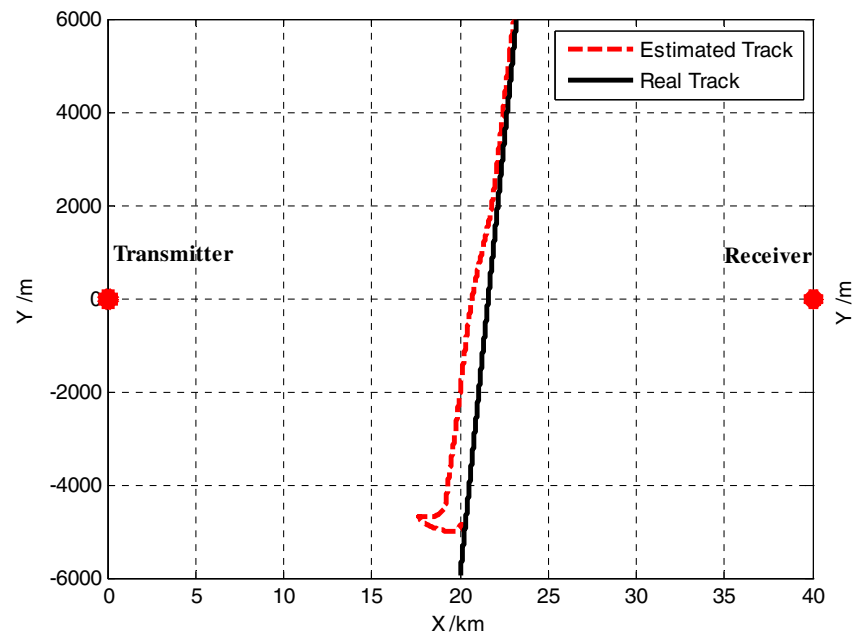

(b)

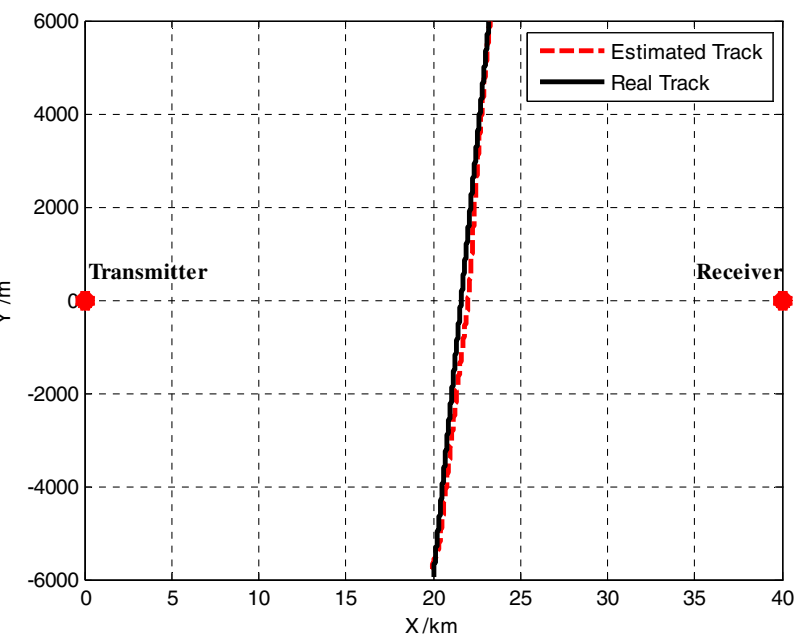

Figure 2 The effect of initial statement estimation errors on target tracking. (a) Tracking results with the initial statement obtained via Gauss-Newton method. (b) Tracking results with the Initial statement obtained via proposed method. 
(a)

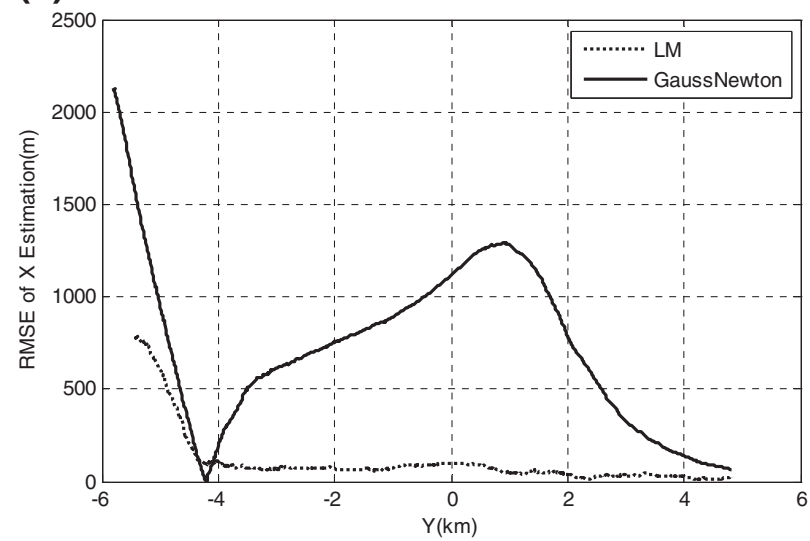

(c)

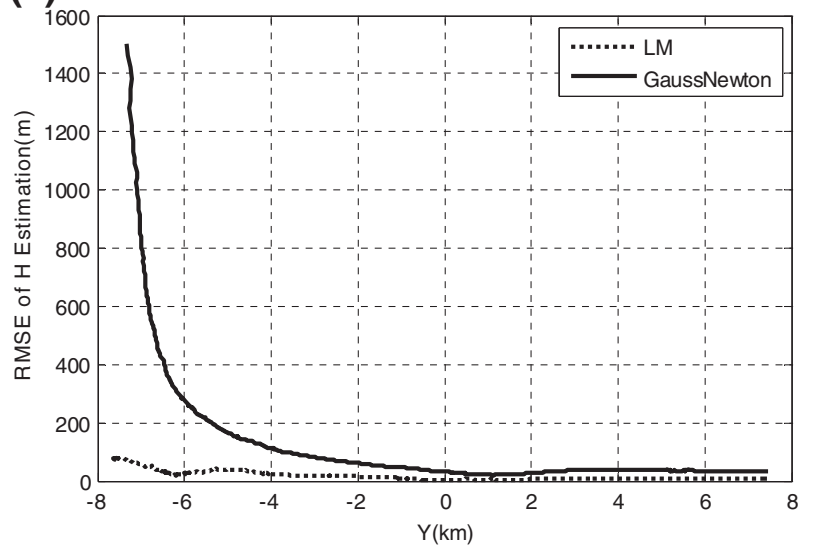

(b)

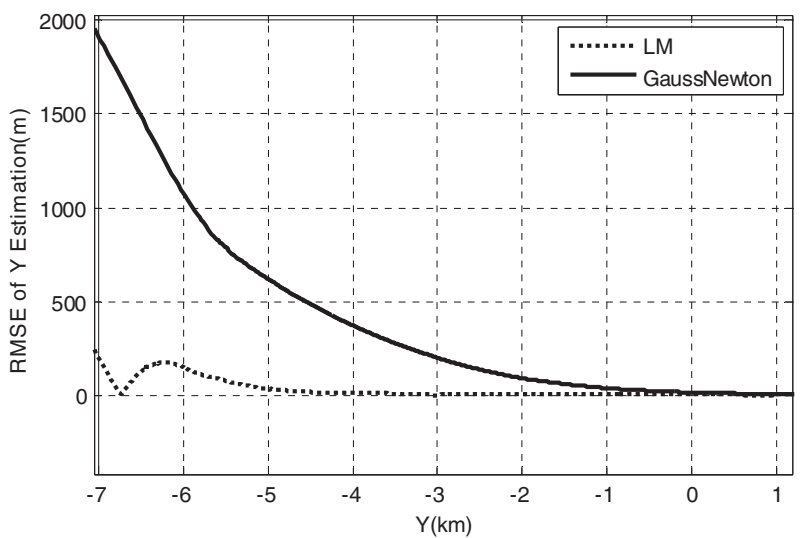

(d)

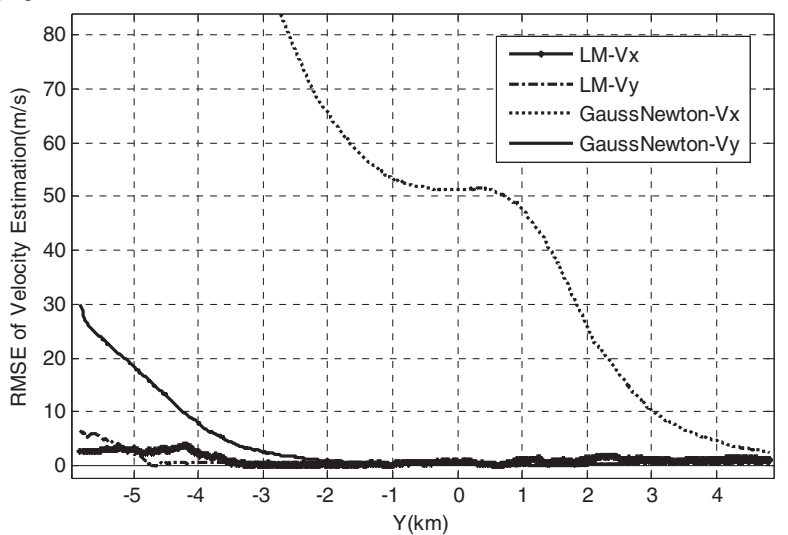

Figure 3 RMSE of target parameters estimation. (a) RMSE of $x$ estimation (b) RMSE of $y$ estimation (c) RMSE of H estimation (d) RMSE of velocity estimation.

Figure 3a-c, RMSE of $X-, Y$-, and $H$-coordinates using initial value obtained by proposed method are all plotted by dotted line, and the ones using initial value obtained by classical method are plotted by solid line. It can be seen that the proposed method gets more precise initial filtering value, which results in the faster convergence and more precise estimation of EKF. While taking the initial state estimated by classical method as the filtering initial value slows down the convergence speed of EKF. In addition, the estimated trajectory has not converged at the time of target crossing baseline. The large RMSE of $V_{x}$ estimation (as shown in Figure 3d) by classical method slows down the convergence of EKF. The initial value of $V_{x}$ is more accurate obtained by the proposed method, which is helpful to achieve rapid filtering convergence.

Then, the overall tracking performances of classical method, new method, and the unscented Kalman filter (UKF) algorithm (taking the initial state obtained by new method as first filtering value) are compared in Figure 4.

Figure 4 shows that the initial state estimation error of classical method is relatively large in the general measurement noise level. In the process of Gauss-Newton iteration, the error is not significantly reduced due to its unstable performance. In the same condition, the initial error of the proposed method is quite small, and gradually decreases until convergence in the follow-up tracking stage. UKF algorithm has a deterioration trend in follow-up tracking.

To compare the computation of different methods, averaging is performed in terms of 100 independent implementations. The time required for one complete filtering of three methods are as follows:

Through analysis of the simulation results, we obtain the following conclusions:

(1)The acquisition of accurate initial state estimation by classical method required very high precision measurement. The initial estimation error significantly increases by the measurement noise.

(2) The proposed method demonstrated its superiority in initial state estimation. In general measurement accuracy, its estimation error is smaller than the classical method's by an order of magnitude. Applied 


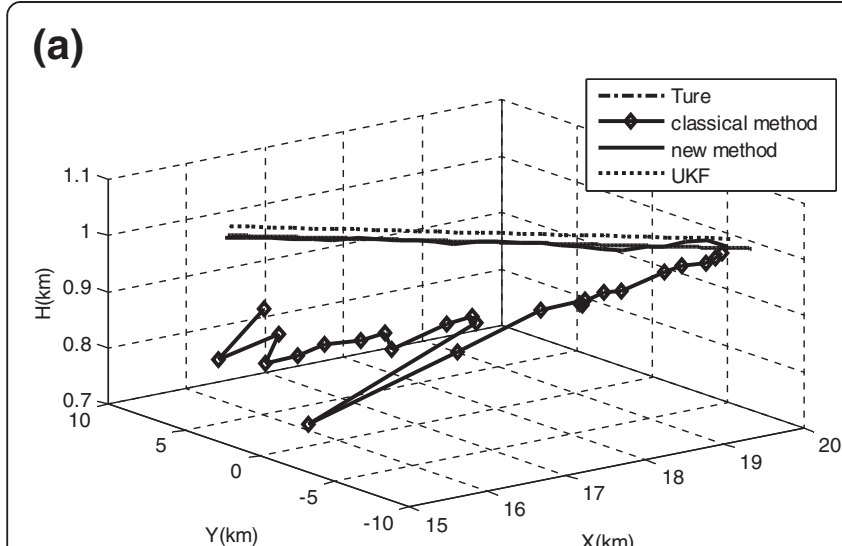

(c)

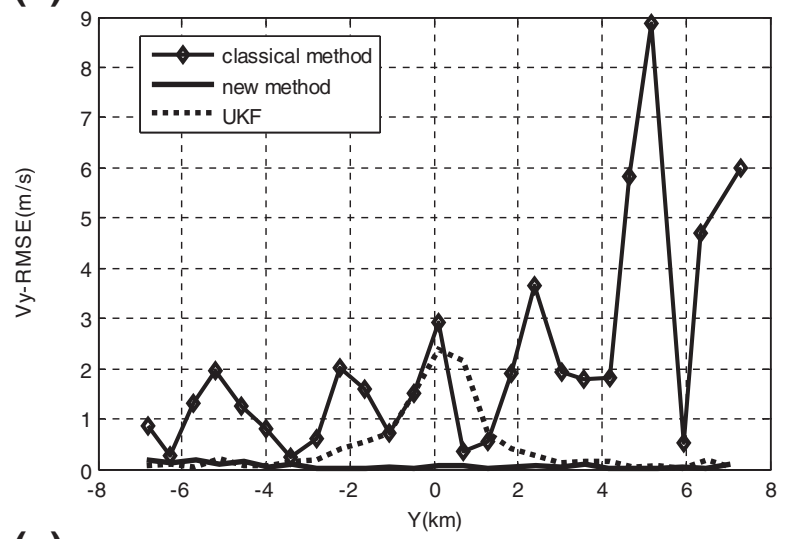

(e)

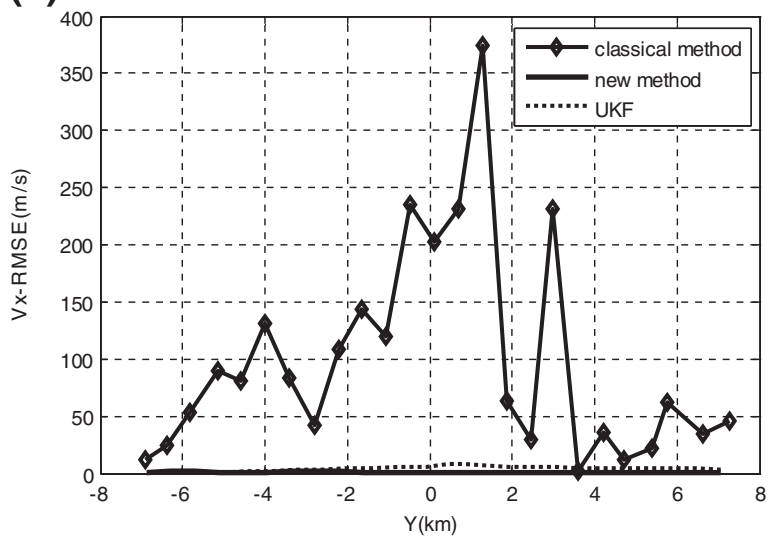

(b)

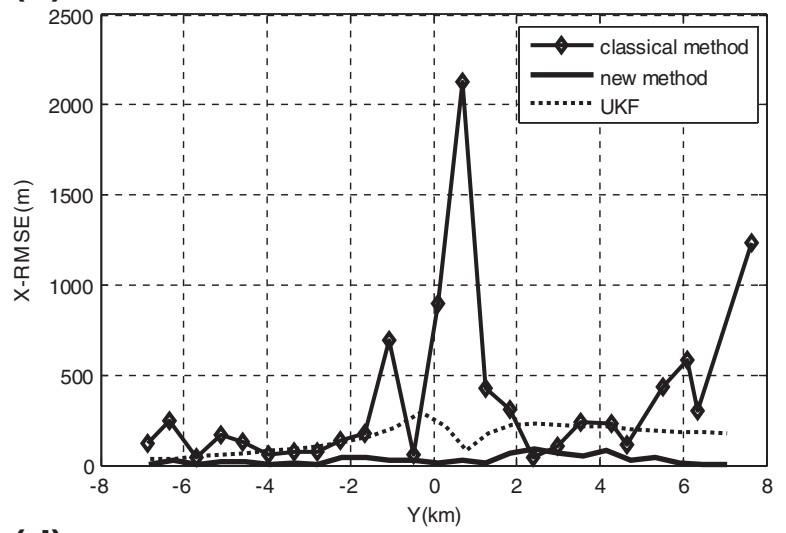

(d)

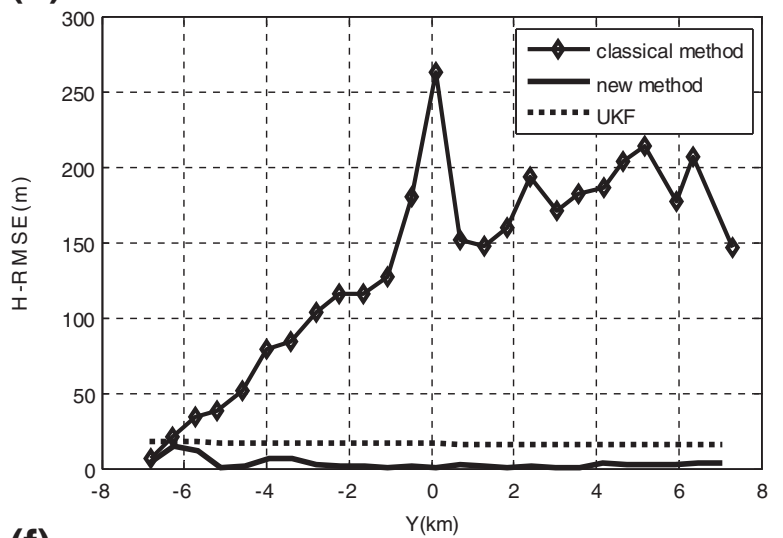

(f)

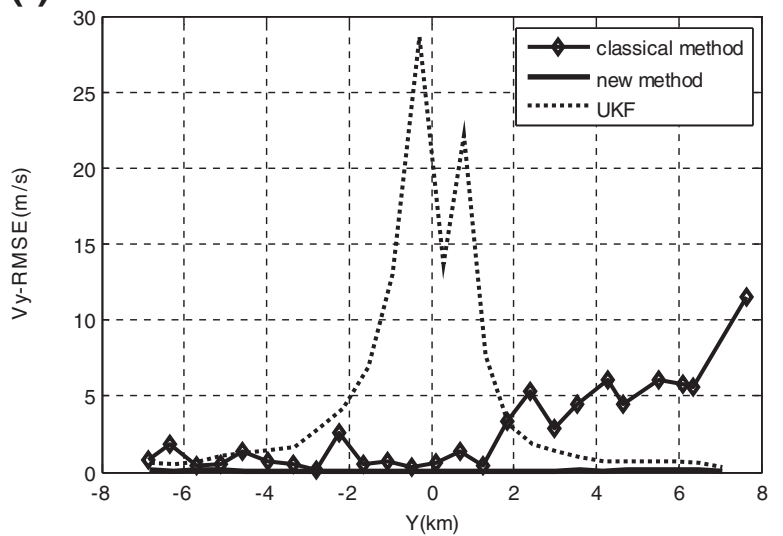

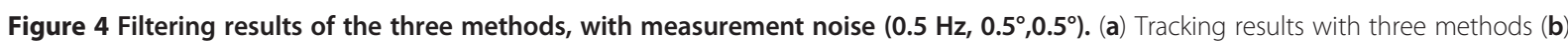
RMSE of $x$ (b) RMSE of $y(\mathbf{c})$ RMSE of $H$ (d) RMSE of Vx (e) RMSE of $V y$.

to EKF, the initial value obtained by the proposed method can effectively improve the convergence speed. Then a stable track is able to be formed before the target crossing baseline.

(3) In general measurement precision, classical method showed non-steady performance and fluctuation of estimation error. Tracking accuracy of UKF algorithm is moderate. But UKF's estimation error has an increasing trend because of its sensitivity to initial error. EKF algorithm can achieve high tracking accuracy and fast convergence with a large tolerance for initial error. Furthermore, its computation is the smallest among three algorithms.

\section{Conclusions}

In this article, an accurate 3D tracking method in forward scatter radar is presented. To solve the problem of filter divergence results from the large initial estimation 
error, an accurate initial state estimation approach based on analytic derivation and LM algorithm is proposed, which can improve the accuracy of initial state estimation without requiring high-precise measurement. For the purpose of reducing the computation of filtering and speeding up the convergence rate, the accurate initial state estimation is combined with EKF algorithm. Then, an accurate $3 \mathrm{D}$ tracking in forward scatter radar is derived and verified by the simulation results.

\section{Competing interests}

The authors declare that they have no competing interests.

\section{Acknowledgment}

This study was supported by the National Natural Science Foundation of China (Grant Nos. 61172177, 61032009, 61225005 and 61120106004).

Received: 3 July 2012 Accepted: 20 February 2013

Published: 2 April 2013

\section{References}

1. NY Willis, Bistatic Radar (Technology Service Corporation (Silver Spring, MD, 1995)

2. AB Blyakhman, Forward scatter bistatic radar, in PIERS Workshop on Advances in Radar Methods (, Italy, 1998), pp. 107-113

3. AB Blyakhman, IA Runova, Forward scatter radiolocation bistatic RCS and target detection, in The Record of the IEEE, International Radar Conference. Massachusetts, USA 1999, 203-208 (1999).

4. AB Blyakhman, AG Ryndyk, SB Sidorov, Forward scatter radar moving object coordinate measurement (The Record of the IEEE 2000 International Radar Conference, Alexandria Virginia, 2000), pp. 678-682.

5. AV Myakinkov, AG Ryndyk, Space-time processing in three-dimensional forward scatter radar, in 4th International Conference on Antenna Theory and Techniques. Ukraine 1, 355-358 (2003).

6. AB Blyakhman, AV Myakinkov, AG Ryndyk, Tracking algorithm for threedimensional bistatic forward scatter radar with weighting of primary measurements, in EURAD Radar Conference, 2005 (, Paris, 2005), pp. 153-156.

7. AV Myakinkov, Optimal detection of high-velocity targets in forward scatter radar, in 5th International Conference on Antenna Theory and Techniques (Ukraine, 2005), pp. 345-347

8. AG Ryndyk, AA Kuzin, AV Myakinkov, AB Blyakhman, Target tracking in forward scatter radar with multi-beam transmitting antenna, in Radar Conference - Surveillance for a Safer World, 2009 (Bordeaux, RADAR, 2009). pp. 1-4

9. DV Nezlin, VI Kostylev, AB Blyakhman, AG Ryndyk, AV Myakinkov, in Bistatic Radar: Principles and Practice, ed. by M Cherniakov (Wiley, UK, 2007)

10. RSA Abdullah, Forward scatter radar for vehicle classification. Phd thesis (School of Electronic, Electrical and Computer Engineering of The University of Birmingham, 2005)

11. $\mathrm{C} \mathrm{Hu}, \mathrm{T}$ Long, $\mathrm{T}$ Zeng, Statistic characteristic analysis of forward scatter surface clutter in bistatic radar. Sci. Chinalnf. Sci. 53(12), 2675-2686 (2010)

12. M Chernicakov, M Salous, R Avdullah et al., Forward scatter radar for ground targets detection and recognition (2nd EMRS DTC Technical Conference, Edinburgh, 2005), pp. A14-A19

13. T Long, C Hu, T Zeng, Physical modeling and spectrum spread analysis of surface clutter in forward scatter radar. Sci. Chinalnf. Sci. 53(11), 2310-2322 (2010)

14. M Cherniakov, RSAR Abdullah, P Jancovic et al., Automatic ground target classification using forward scatter radar. IEE Proc. Radar Sonar \& Navigation. 153(5), 427-437 (2006)

15. T Long, C Hu, M Cherniakov, Ground moving target signal model and power calculation in forward scatter micro radar. Sci. China Ser FInf. Sci 52(9), 1704-1714 (2009).

16. T Zeng, L X-I, C Hu, Investigation on accurate signal modeling and imaging of ground moving target in forward scattering radar. IET Radar Sonar Navigat 5(8), 862-870 (2011)

17. C Hu, Theory and signal processing methods of short baseline forward scatter radar system, PhD Thesis (Beijing Institute of Technology, 2009), p. 7
18. TL Song, JL Speyer, A stochastic analysis of a modified gain extended Kalman filter with application to estimation with bearing only measurements. IEEE Trans. Autom. Control 30(10), 940-949 (1985)

19. K Levenberg, A method for the solution of certain nonlinear problems in least squares. Q. Appl. Math. 2(2), 164-168 (1944)

20. D Marquardt, An algorithm for the least-squares estimation of nonlinear parameters. SIAM J. Appl. Math. 11(2), 431-441 (1963)

doi:10.1186/1687-6180-2013-66

Cite this article as: Hu et al:: Accurate three-dimensional tracking method in bistatic forward scatter radar. EURASIP Journal on Advances in Signal Processing 2013 2013:66.

\section{Submit your manuscript to a SpringerOpen ${ }^{\odot}$ journal and benefit from:}

- Convenient online submission

- Rigorous peer review

- Immediate publication on acceptance

- Open access: articles freely available online

- High visibility within the field

- Retaining the copyright to your article

Submit your next manuscript at springeropen.com 